\title{
Capabilities evaluation of spaceborne scientific equipment for geophysical applications
}

\author{
Ivan Kaloshin ${ }^{1, *}$, Vladimir Kuznetsov ${ }^{1}$, Vladimir Skripachev ${ }^{1}$, and Irina Surovceva ${ }^{1}$ \\ ${ }^{1}$ Moscow Technological University (MIREA), 119454 Moscow, Russia
}

\begin{abstract}
The physical phenomena such as earthquake precursors recorded by on-board scientific equipment are examined in details. The selection method for geophysical satellite data of informative sources is discussed. The on-board scientific instruments complex application possibility for seismic activity diagnosis is shown. The development prospects of earthquakes precursors space monitoring system are defined.
\end{abstract}

\section{Introduction}

Satellites on-board scientific equipment provide information about various processes and phenomena on the Earth. Earthquakes are one of the most dangerous phenomena, due to the suddenness of their occurrence.

The importance of earthquakes precursors monitoring with on-board scientific equipment is determined by strict requirements for data acquisition operability, the earth coverage magnitude and data set sufficiency on their completeness (informativeness).

Strong crustal earthquakes formation process is accompanied by various precursors appearance: the Earth's crust deformation, electromagnetic field variations, anomalous cloud formations, etc. The main objective to identify earthquakes precursors from the space is the diagnosis of space and time environment parameters in seismically active regions.

We shall consider physical phenomena associated with seismic activity and recorded by on-board scientific instruments.

\section{Physical phenomena and on-board instruments}

The noise increase in the frequency range 100-500 Hz was recorded 14 hours before the earthquake from low orbit satellites over the epicenter of the earthquake with $\mathrm{M}>5.4$. The abnormal increase of the low-frequency radio waves $(0.1-15 \mathrm{kHz})$ intensity at the moment of earthquakes was recorded by magnetic and electric antennas [1].

According to [2] a correlation of the seismic activity with captured by the geomagnetic field particle fluxes variations was found. Spatial-temporal parameters analysis showed the rapid increase of the particles flux before the earthquake. This feature was formed during the phenomena distribution 2.5 hours before the earthquake strike.

\footnotetext{
Corresponding author: kaloshin@mirea.ru
} 
It is known that the earthquakes are accompanied by large thermal anomalies development that occur 2-3 weeks before the event and last 1-2 days after it. Such anomalies intensity is typically $3-4^{\circ} \mathrm{C}$, with not only the earth's surface heating but the air heating above the epicenter as well [3].

Analysis of the cloud cover on the satellite images have demonstrated the presence of anomalous zones in the visible and infrared regions of the electromagnetic spectrum. The further studies have shown that the anomalous zone is best revealed in the fragments of space images of the earth surface areas sizes from $200 \times 200$ to $300 \times 300 \mathrm{~km}$ [3].

There are basic requirements for all on-board scientific instruments: maximum efficiency; dimensions, weight characteristics and electromagnetic compatibility; to satisfy other requirements for mechanical, thermal, electrical and other satellites hardware characteristics. Now we shall briefly consider some scientific instruments of satellites for geophysical applications.

Ionosond is one of the effective means of scientific equipment to solve the problem of seismic activity precursor's detection. It spends pulse sounding of the ionosphere by providing timely information about its condition, including the critical frequency F2 layer ionospheric electron concentration. Use experience of such devices (e.g. IS-338) shows the possibility of operating on more than 300 fixed frequencies in the range of $0.3 \ldots 15.95$ $\mathrm{MHz}$ for altitudes from $400 \mathrm{~km}$ to $1,000 \mathrm{~km}$.

The detector of protons and electrons with the energy range of $20 \mathrm{keV}-2 \mathrm{MeV}$ is widely used in the low orbit satellites. Such detector for measuring of the differential spectra of both electronic and ionic (proton) components of geoactive corpuscular radiation has been successfully tested on board the «Meteor» satellites.

Now some words about the global navigation satellite systems GLONASS/GPS signals receivers. These devices are essential for ionosphere radio tomography from low orbit satellites. It is also useful for radio occultation measurements. Those receivers are able to detect ionospheric disturbances.

To register anomalies in the ULF/VLF range it is necessary to install the instruments that allow to detect the signals appearing in the ionosphere before the earthquake. The instrument must work in the range of $10 \mathrm{~Hz}$ to $20 \mathrm{kHz}$, as the majority of the available experimental data has been obtained in this frequency range.

Functionally the radiometers consist of multiple spectral channels, both visible and infrared rangeы and provide images of the Earth surface. The processing images allow to obtain a number of precursors such as temperature anomalies, cloud cover anomalies and other meteorological precursors. As an example, it could be the radiometer AVHRR and radiometer MSU. In addition, these phenomena could be detected by passive microwave radiometers MTVZA with spatial resolution 20-100 km.

Mass spectrometers, which operation is based on ionized particles separation by their masses under the influence of magnetic and electric fields that allow the measurement of ionized and neutral components of ionospheric plasma. These instruments must provide the measurement of relative content of ion concentrations key component of the ionospheric plasma in the range of 1-65 mass amu with a resolution of 10-15 amu.

To estimate opportunities onboard scientific equipment of perspective satellites for short-term forecasting of earthquakes, it is necessary to choose the most informative sources of satellite data. To do that we have combined the expert information and paired comparisons methods. Table 1 shows the main sources of predictive information. The proposed method is based on the expert information processing. 


\section{Expert information processing technique}

Each expert has filled in two matrices on the earthquake precursor's relevance and the spaceborne instruments feasibility. The count of experts $N=6$. The experts were employees of IKI RAS, MIREA, «Russian Space Systems» JSC, IRE RAS.

Weights calculation in accordance with the values, which ranged the matrices elements, is an iterative process [4]:

$$
p^{t}=\mathrm{A} p^{t-1}
$$

where $p^{0}=(1,1, \ldots, 1)^{\prime}$.

During the iteration it is possible to obtain large weight values. To avoid that, the components of the vector $p^{t}$ must be normalized at each step of iteration by following value:

$$
\lambda^{t}=\Sigma_{i} p_{i}^{t}=\Sigma_{i} \Sigma_{j} a_{i j} p_{j}^{t-1}
$$

According to the normalized factor the weights calculation procedure is:

$$
P^{t}=\mathrm{A} p^{t-1} / \lambda^{t}
$$

Table 1. Main information sources of earthquake precursor.

\begin{tabular}{|c|c|}
\hline Parameter & Earthquake precursor information \\
\hline $\mathrm{A}_{1}$ & Surface temperature \\
\hline $\mathrm{A}_{2}$ & Temperature and humidity vertical profile \\
\hline $\mathrm{A}_{3}$ & Cloud cover anomalies \\
\hline $\mathrm{A}_{4}$ & Vapor flow \\
\hline $\mathrm{A}_{5}$ & Upper-level wind (9-12 km) \\
\hline $\mathrm{A}_{6}$ & Thfrared radiation flows at the height of the upper clouds edge \\
\hline $\mathrm{A}_{7}$ & Thermal anomalies \\
\hline $\mathrm{A}_{8}$ & temperature), including electron concentration vertical profile \\
\hline $\mathrm{A}_{9}$ & Ion composition \\
\hline $\mathrm{A}_{10}$ & ULF/VLF-emissions \\
\hline $\mathrm{A}_{11}$ & Electromagnetic fields \\
\hline $\mathrm{A}_{12}$ & Particles fluxes with energy spectrums \\
\hline
\end{tabular}

The computational process continues until the moment when the weighs obtained in successive iterations slightly differ from each other, i.e.

$$
\max \left|p_{i}^{t}-p_{i}^{t-1}\right|<\varepsilon
$$

where $\varepsilon=0.005$-calculation accuracy.

The priorities vector components estimation has been made separately. The polling of experts involved in the examination was carried out in view of the fact that their competence was the same. This procedure was repeated for each expert data processing. The final group score was obtained by averaging the individual assessments values. 
Ranking was used to evaluate the significance of the experts polling results. Each value $A_{1}, \ldots, A_{12}$ was assigned a rank - serial number in the ascending order.

To determine the strength of relationship between the ranks of the parameters $A_{1}, \ldots, A_{12}$ a coefficient of concordance was determined. Since the ranks are disconnected, then the coefficient of concordance is defined as:

$$
W=12 S /\left[m^{2}\left(n^{3}-n\right)\right]
$$

where $m=2$ - number of properties (relevance of the earthquake precursors, feasibility of the spaceborne instruments); $n=12$ - number of matrices parameters $A_{1}, \ldots, A_{12}$;

$$
S=\Sigma_{m}{ }^{n}\left(\sum_{j}^{m} R_{i j}\right)^{2}-\left(\sum_{i=1}{ }^{n} \sum_{j=1}{ }^{m} R_{i j}\right)^{2} / n
$$

where $R_{i j}$ - rank of $i$ parameter of $j$ property.

The coefficient of concordance significance was estimated by means of Chi-squared statistic. Estimated value $\chi_{\mathrm{c}}^{2}$ in the absence of connected ranks is determined by the formula:

$$
\chi_{\mathrm{c}}^{2}=12 S /[m n(n+1)]
$$

As the defined value $\chi_{\mathrm{c}}{ }^{2}=6.90$ exceed the table value $\chi_{\mathrm{tab}}{ }^{2}=4.57$, with given significant level $\alpha=0.95$ and degrees of freedom $d f=11$, such coefficient of concordance becomes significant. Having applied the relative normalizing by maximum value, the following vector of priorities was obtained; the results are shown in Table 2.

To identify the priority informative sources of satellite data, the threshold with weighted average value $L=0.7$ was used (Figure 1).

Table 2. Normalized priorities vector.

\begin{tabular}{|c|c|c|c|c|c|c|c|c|c|c|c|}
\hline $\mathbf{A}_{\mathbf{1}}$ & $\mathbf{A}_{\mathbf{2}}$ & $\mathbf{A}_{\mathbf{3}}$ & $\mathbf{A}_{\mathbf{4}}$ & $\mathbf{A}_{\mathbf{5}}$ & $\mathbf{A}_{\mathbf{6}}$ & $\mathbf{A}_{\mathbf{7}}$ & $\mathbf{A}_{\mathbf{8}}$ & $\mathbf{A}_{\mathbf{9}}$ & $\mathbf{A}_{\mathbf{1 0}}$ & $\mathbf{A}_{\mathbf{1 1}}$ & $\mathbf{A}_{\mathbf{1 2}}$ \\
\hline 0.684 & 0.658 & 0.776 & 0.387 & 0.338 & 0.365 & 0.962 & 1.000 & 0.779 & 0.889 & 0.630 & 0.748 \\
\hline
\end{tabular}

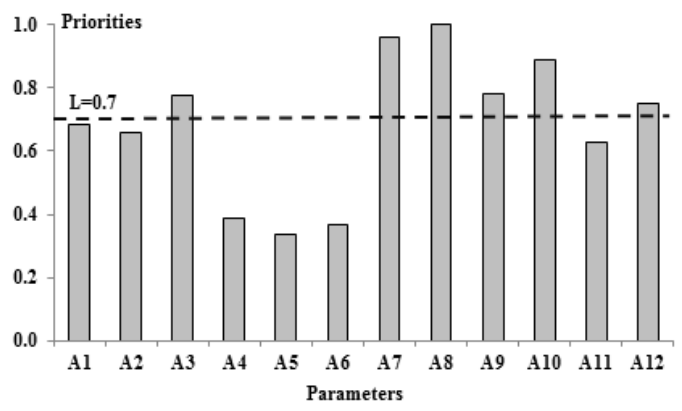

Fig. 1. Histogram of normalized priorities vector.

\section{Results}

One can see that parameters $A_{3}, A_{7}, A_{8}, A_{9}, A_{10}, A_{12}$ have the highest priority for geophysical monitoring. According to the expert estimation results in order to detect earthquake precursors it is advisable to diagnose identified parameters and to have on-board scientific equipment for the registration following parameters: local disturbances of ionospheric plasma (electrons concentration, electrons and ions temperature), including electron 
concentration vertical profile; thermal anomalies; ULF/VLF-emissions; ion composition; cloud cover anomalies; particles fluxes energy spectrums.

Thus, the selecting method of the most informative sources of satellite data received by on-board devices should be based on the satellite dimensions, orbital constellation and above requirements. The main tools are:

- signals receiver of global navigation satellite systems (dual-frequency with a measurement rate of not lower than $20 \mathrm{~Hz}$ ) for transionospheric sensing;

- ULF/VLF complex with $0.02-20 \mathrm{kHz}$ work range;

- IR camera with spatial resolution of $15 \ldots 250 \mathrm{~m}$ and resolution $5 \mathrm{~K}$;

- high-energy particle detector with a measuring range from 3 to $100 \mathrm{MeV}$ for fluxes of energetic particles generated as a result of seismic activity.

\section{Conclusion}

The results obtained allow to estimate the optimum composition and technical characteristics of satellite on-board scientific equipment for sensing earthquake precursors, and to determine the prospects of the space seismic activity monitoring system development.

The following can be recommended as an important addition to satellite data for a forecast earthquake monitoring system: ground-based signals receivers of global navigation satellite systems (GLONASS, GPS) and ground-based VLF-receivers, which allow to observe seismo-ionospheric disturbances.

\section{References}

1. M. Parrot., ASR 15(11), 27 (1995)

2. V. Pustovetov, E. Ginzburg, A. Malyshev, I. Proshkina, Cosmic Research 31(3), 123 (1993)

3. A. Tronin, M. Hayakawa, O. Molchanov, J. Geodynamics 33, 519 (2002)

4. V. Davnis, V. Tinyakova, Prognoznye modeli ekspertnykh predpochteniy (VSU, Voronezh, 2005) (in Russian) 